\title{
Relevância da comunicação de más notícias pelo profissional da saúde de maneira adequada: revisão narrativa
}

The relevance of appropriate communication of bad news by the health care professional: narrative review

La relevancia de la comunicación adecuada de las malas noticias por parte de los profesionales sanitarios: revisión narrativa

Patricia Dupont $^{1 *}$, Giorgia Polati El-Dine ${ }^{1}$, Siegrid Kurzawa Zwiener dos Santos ${ }^{2}$.

\section{RESUMO}

Objetivo: Expor as dificuldades enfrentadas pelo médico em transmitir uma má notícia ao paciente. Revisão bibliográfica: Estudos mostram que os médicos que utilizam como base o protocolo SPIKES (o protocolo mais utilizado atualmente) na hora da comunicação de más notícias, encontram menos dificuldades durante essa ação. Evidencia-se que esse tipo de diálogo é inevitável durante o período de atuação do profissional de saúde, sendo assim, este necessita desenvolver habilidades que visam transmitir diagnósticos e prognósticos difíceis de forma adequada e empática. A comunicação é um processo dinâmico e aberto. No quesito saúde, engloba desde a comunicação de notícias, até a obtenção de informações. "Má notícia" é considerada como toda a informação que gera uma mudança extrema e negativa na vida do paciente e de seus familiares na visão do seu futuro. Considerações finais: Diante de tais motivações encontradas, percebe-se que há um déficit na grade curricular das faculdades de medicina em relação ao âmbito da comunicação de notícias difíceis aos pacientes e seus familiares, visto que muitos médicos encontram dificuldades nessa tarefa.

Palavras-chave: Relação médico-paciente, Comunicação de más notícias, Protocolo SPIKES, Empatia.

\begin{abstract}
Objective: To expose the difficulties faced by physicians in transmitting bad news to patients. Bibliography review: Studies show that physicians who use the SPIKES protocol (the most used protocol today) as a basis when communicating bad news, find fewer difficulties during this action. It is evident that this kind of dialogue is inevitable during the period of the health professional's performance, so he/she needs to develop skills that aim to transmit difficult diagnoses and prognoses in an adequate and empathetic way. Communication is a dynamic and open process. In health care, it ranges from communicating news to obtaining information. "Bad news" is considered as all the information that generates an extreme and negative change in the life of the patient and his family in the vision of his future. Final considerations: In view of these motivations found, it can be seen that there is a deficit in the curricula of medical schools in relation to the communication of difficult news to patients and their families, since many physicians find this task difficult.
\end{abstract}

Keywords: Doctor-patient relationship, Communication of bad news, SPIKES protocol, Empathy.

1 Universidade do Contestado, Mafra - SC. *E-mail: patriciadupont77@gmail.com

2 Universidade Federal do Paraná (UFPR), Curitiba - PR. 


\section{RESUMEN}

Objetivo: Exponer las dificultades a las que se enfrentan los médicos a la hora de transmitir malas noticias a los pacientes. Revisión bibliográfica: Los estudios demuestran que los médicos que utilizan el protocolo SPIKES (el más utilizado actualmente) como base a la hora de comunicar malas noticias, encuentran menos dificultades durante esta acción. Es evidente que este tipo de diálogo es inevitable durante el periodo de actuación del profesional sanitario, por lo que necesita desarrollar habilidades que tengan como objetivo transmitir diagnósticos y pronósticos difíciles de forma adecuada y empática. La comunicación es un proceso dinámico y abierto. En el ámbito de la salud, abarca desde la comunicación de noticias hasta la obtención de información. Se consideran "malas noticias" todas aquellas informaciones que generan un cambio extremo y negativo en la vida del paciente y su familia en la visión de su futuro. Consideraciones finales: Frente a estas motivaciones encontradas, se percibe que existe un déficit en el currículo de las facultades de medicina en relación a la comunicación de noticias difíciles a los pacientes y sus familias, ya que muchos médicos encuentran esta tarea difícil.

Palabras clave: Relación médico-paciente, Comunicación de malas noticias, Protocolo SPIKES, Empatía.

\section{INTRODUÇÃO}

Para ter boa relação entre profissional de saúde e seus pacientes é muito importante haver uma comunicação adequada com os pacientes e familiares. Hoje, no Brasil, percebe-se um aumento na dificuldade enfrentada pelos médicos na comunicação de más notícias. A comunicação constitui em recurso fundamental no âmbito da saúde, pois fortalece laços, desenvolve a autonomia do paciente e melhora o vínculo de confiança, através da relação médico-paciente. No entanto, comunicar não é considerado uma ação simples ou fácil, pois relaciona-se a crenças, valores e princípios que podem interferir na transmissão das informações (KOCH CL, et al., 2017).

Entretanto, a linguagem utilizada para comunicar a família e o paciente deve ser acessível, cabendo ao médico se certificar de que o enfermo e os familiares compreenderam todas as informações transmitidas, sem obrigatoriedade de que o consentimento seja reduzido a termo, ou seja, registrado por escrito (GAZZOLA LPL, et al., 2020).

Porém, existe um protocolo que auxilia muito na hora de comunicar o doente e sua família, o chamado protocolo SPIKES (setting up, perception, invitation, knowledge, emotions, strategy), que nada mais é que um mnemônico de seis passos que pode proporcionar mais segurança ao médico na hora de dialogar com o paciente e que mostra quatro metas principais: entender o que 0 paciente e seus familiares estão compreendendo da situação como um todo (auxilia o médico a saber por onde iniciar), oferecer as informações de modo com o que o paciente e sua família consigam lidar com a situação, acolher qualquer reação que possa vir a acontecer e, por último, ter um plano terapêutico ou paliativo (CRUZ CO e RIERA R, 2016).

Nesse contexto, a comunicação de más notícias é uma das atividades exercidas pelos profissionais da saúde, sendo a mais complexa do processo do tratamento por conta da relação de proximidade com a morte. Porém, mesmo que a medicina está avançando gradativamente em questão de tecnologia, a inclusão da prática de habilidades comunicativas, principalmente no momento de comunicar más notícias, relacionadas na formação clínica, é defendida pelos profissionais como uma experiência que deveria ser obrigatória, mas é, na verdade, facultativa e pouco valorizada, sendo perceptível a dificuldade de alguns profissionais no momento da comunicação de más notícias aos pacientes e seus familiares (SANTOS GFATF, et al., 2020; OSTERMANN A, et al., 2017).

Diante do exposto, o presente artigo trata-se de um ensaio teórico, de base descritiva, com o objetivo de propor uma reflexão acerca da temática que envolve expor as dificuldades enfrentadas pelo médico em transmitir uma má notícia ao paciente, justificando-se pela relevância do tema no cotidiano. 


\section{REVISÃO BIBLIOGRÁFICA}

\section{Comunicação de más notícias}

Na sociedade atual, o assunto "morte" é visto com preconceito, sendo representado como o mais absoluto horror, com intenso sofrimento. Entretanto, comunicar uma notícia difícil é considerado um desafio estressante e difícil para os médicos, sendo assim, muitos evitam a transmissão dessa notícia ou a realizam de maneira incorreta (KOCH C, et al., 2017; MONTEIRO D e QUINTANA A, 2016).

Porém, comunicar a morte requer disponibilidade e preparo emocional dos profissionais de saúde. Ser o precursor de notícias tristes e irreversíveis, tem como primeira dificuldade, o enfrentamento do sentimento de fracasso emocional, que abala emocionalmente quem transmite a mensagem. A experiência da morte de outros é considerada indiretamente a própria experiência da morte. Esta situação ocorre com todas as pessoas que estão acompanhando o processo de morte e adoecimento e é particularmente dolorosa para os profissionais de saúde, porque implica que eles estão constantemente refletindo sobre suas atitudes pessoais e profissionais com vidas limitadas (KOCH CL, et al., 2017).

Sabe-se que comunicar notícias de diagnóstico em um ambiente hospitalar é um evento de impacto potencial para todos os envolvidos. No entanto, embora o aspecto médico-paciente permaneça inalterado, essa tarefa raramente é ensinada na faculdade e no treinamento clínico, sendo essa a segunda dificuldade enfrentada pelo medico ao comunicar má notícia (OSTERMANN AC, et al., 2017).

Todavia, os profissionais aprendem a relatar más notícias por acerto e erro ou também pela observação de colegas que são mais experientes, mas isso não garante uma comunicação eficaz e de boa qualidade, que não haja consequências, e não há evidências suficientes de que as habilidades dos profissionais irão se desenvolver ao longo do tempo de forma competente (FREIBERGER MH, et al., 2019).

Por outro lado, entende-se que, comunicar más notícias, principalmente no diagnóstico de uma doença incurável, é um assunto delicado para os profissionais, pois as emoções manifestadas pelos pacientes muitas vezes são difíceis de serem controladas, sendo essa, a terceira adversidade encontrada pelo médico. Entretanto, a capacidade de lidar com essa situação não é inata nem dotada por Deus, mas pode ser obtida por meio de informação e treinamento, para que o impacto negativo no paciente seja minimizado. Por isso, é necessário adaptar aos cursos de medicina o estudo da comunicação, especialmente para saber lidar com pacientes que possuem comorbidades (FREIBERGER MH, et al., 2019).

Ressalta-se, também, outro quesito importante na comunicação das más notícias: a empatia. $O$ comportamento de empatia, nas relações interpessoais, mostra a necessidade contemporânea de uma maior compreensão das relações com as outras pessoas, o que está intimamente relacionado a ter uma boa relação médico-paciente. Ao demonstrar empatia, o profissional apoia o paciente e à família no enfrentamento do momento difícil, condição considerada necessária para a criação de um ambiente emocionalmente confortável. A empatia estabelece relação em que não só o que é dito afeta a transmissão da informação, porque o tom e o ritmo da voz, o gesto, o toque e o silêncio também vinculam sinergicamente pensamentos acolhedores e aconchegantes (KOCH CL, et al., 2017).

Sendo assim, a comunicação é muito mais complexa do que uma simples troca de palavras entre o médico e o seu paciente, é considerado um processo dinâmico e aberto. É compreendida como uma ferramenta muito importante do plano terapêutico, porque além de garantir a autonomia do paciente, fortalece a relação de confiança entre o paciente, sua família e o profissional de saúde (VOGUEL KP, et al., 2019).

\section{Protocolo spikes}

Entre as diversas aptidões clínicas necessárias para um trabalho bem-sucedido em saúde, a comunicação entre médico e paciente é ressaltada, pois fornece acolhimento, diálogo e entendimento a ambos. Desta forma, o uso de protocolos para a comunicação de más notícias é usado como guia para a prática, sendo muito utilizado e reconhecido por profissionais da saúde, pois ajuda a superar dificuldades que podem surgir durante a ação. Um dos protocolos utilizados seria o protocolo SPIKES, que foi desenvolvido por Robert 
Buckman, um médico oncologista, que foi pioneiro nos estudos relacionados à comunicação de más notícias, que relata estágios importantes a se considerar durante a comunicação (KOCH CL, et al., 2017).

Buckman sugere que comunicar notícias difíceis aos pacientes é uma habilidade, não um "dom divino", e que todos podem ensinar e compreender que esta é uma parte importante do cuidado com os pacientes. $O$ protocolo é um acordo que contém seis etapas estratégicas para orientar os profissionais de saúde na comunicação de notícias difíceis, sendo esta uma das formas mais eficazes e didáticas de veicular más notícias (FREIBERGER M H, et al., 2019; KOCH C, et al., 2017; CAMPOS CACA, et al., 2017).

Dessa forma, o protocolo SPIKES é composto por: Setting, que é a primeira etapa, e inclui uma abordagem inicial adequada do paciente e a forma como o transmissor da notícia ajeita o ambiente, preservando sempre o sigilo e a privacidade, acolhendo de forma adequada o paciente e seus familiares. Em segundo momento a perception, que analisa o quanto o paciente já sabe sobre sua doença ou condição de saúde e futuras possibilidades. E como terceira fase há o invitation, sendo um convite para descobrir quanto o paciente deseja saber sobre a sua condição (GAZZOLA LPL, et al., 2020).

Além disso, há o knowledge na quarta etapa, seria passar o conhecimento, compartilhar a informação, comunicar a notícia propriamente dita, que deve ser transmitida com linguagem compreensível para 0 paciente e familiares, de forma realista, empática e afetuosa. Já as emotions, na quinta etapa, seria a empatia do médico para responder e amparar as emoções do paciente, qualquer que seja sua reação a partir da recepção da notícia. Entretanto, a strategy nessa sexta e última etapa, são discutidas estratégias que podem ser usadas para conduzir o planejamento terapêutico do paciente e diminuir a dor e a ansiedade em relação a notícia recebida (GAZZOLA LPL, et al., 2020).

Constitui-se de suma importância deixar claro para o paciente após a transmissão da má notícia que ele não será desamparado, que existe um plano ou tratamento paliativo, que poderá reduzir sua dor e sofrimento (CRUZ CO e RIERA R, 2016).

Para o médico, uma das etapas mais difíceis é a quinta fase do protocolo de SPIKES, quando ele deve apoiar a resposta emocional mais diversa do paciente e combiná-la com suas próprias emoções e experiência. Nesse estágio, é comum que os pacientes fiquem com raiva do médico, o portador de más notícias, e pelo menos inicialmente o considerem um traidor, e não um aliado. Para esse tipo de resposta, empatia, verdade e linguagem são os mais básicos, pois é necessário garantir que o paciente compreenda a doença para participar do processo de tomada de decisão comum. Sendo assim, percebe-se que comunicar más notícias não é um trabalho fácil. A finalidade do protocolo SPIKES é, de alguma maneira, organizar este momento, ajudando médicos e pacientes a manter uma comunicação fácil, clara e aberta (GAZZOLA LPL, et al., 2020; CRUZ CO e RIERA R, 2016).

\section{Comunicação de más notícias durante a gestação e período neonatal}

Sabe-se que, os neonatos e pediátrico apresentam diferenças em relação aos centros de atendimento hospitalares. A quarta dificuldade, dá-se quando profissionais da saúde manuseia com a vida ou até mesmo a morte no cenário infantil. Ou seja, necessitam conviver com o sofrimento dos familiares e frustrações, enfrentando a exigência de constantemente comunicar situações constrangedoras e tristes, além da aflição provocada por essas tarefas (KOCH CL, et al., 2017).

Entretanto, a certificação de que o feto apresenta alguma malformação ainda intraútero, condiz como má notícia, e sua comunicação à gestante e familiares gera conflitos éticos relacionados à medicina fetal e neonatologia. A frustação na espera dos pais quanto ao filho tão desejado provoca frustrações, e a forma de processar a notícia pode ser diretamente influenciada pela forma como a notícia é comunicada pela equipe a família. Para os casos em que a criança nasce com vida, a sugestão de iniciar cuidados paliativos também deve ser compartilhada, dando assim, uma esperança a mais para a família (GAZZOLA LPL, et al., 2020).

Sabe-se que, com uma ideal comunicação em saúde, é possível apoiar e minimizar os sentimentos de dor, desamparo, frustração, estresse e ansiedade vivenciados pela família da criança, quando se refere à más notícias. Dessa forma, deve ser transmitido aos pais todas as circunstâncias e desfechos possíveis, como 
provável morte intrauterina ou sobrevivência por tempo limitado após o nascimento, assim como o resultado da gestação ou o momento do óbito do feto/criança. Durante o auxílio, os pais devem ser informados dos riscos de uma futura gestação, e o modo como ocorre a interpretação dos mesmos pode interferir a escolha quanto à situação atual (KOCH C, et al., 2017; GAZZOLA L, et al., 2020).

Um estudo realizado mostra as visões otimistas ofertadas em um aconselhamento genético em que há a comunicação de que o feto tem diagnóstico de síndrome de Down, juntamente a uma cardiopatia congênita. Consequente à notícia, a gestante chora na primeira consulta, comunica estar com depressão e então foi encaminhada para o setor de psiquiatria do mesmo hospital. Nesse caso, o médico deve se posicionar de forma empática para a paciente e avaliar positivamente o fato de existir muito o que fazer nessa situação. Oferecer possibilidades a mãe, como por exemplo a sugestão de que há cirurgia para corrigir a cardiopatia que o feto apresenta (OSTERMANN AC, et al., 2017).

Contudo, a gestação é marcada por expectativas para o futuro embasadas em projeto idealizado de parentalidade. Muito antes do término do desenvolvimento, o bebê já existe para a mulher, que se imagina mãe e constrói um pensamento maravilhoso com o futuro filho, então a informação de que seu bebê pode vir ao mundo com malformação, deve ser transmitida aos familiares com muito cuidado e de forma empática. Sendo assim, percebe-se novamente como a comunicação é essencial na formação de profissionais e nas práticas de trabalho em equipe, buscando consensos para construção de projetos que beneficiam os pacientes (GAZZOLA L, et al., 2020; CAMPOS CACA, et al., 2017).

\section{Comunicação de más notícias em câncer e doenças terminais}

O momento da má notícia é quando o profissional deve parar, refletir e se preparar para conversar com os familiares, o que não quer dizer uma encenação, mas compreender a história da saúde e da doença e organizar seu tempo e lugar para acolher esta família. Diante da morte, esse tipo de diálogo é inevitável, pois os profissionais devem se questionar sobre a melhor forma ou como passar essas informações para a família observando as peculiaridades de cada local e de cada pessoa (LYSAKOWSKI S, et al., 2020).

Todavia, um momento em que as más notícias necessitam ser comunicadas é no caso do câncer, que é considerado um problema de saúde pública e agora está aumentando significativamente tanto nos países desenvolvidos quanto nos em desenvolvimento. Portanto, considera-se necessário afirmar que em oncologia, tanto na fase inicial da doença quanto na fase final da doença, notícias difíceis estão se espalhando constantemente, o que exige que os médicos oncologistas desenvolvam habilidades que visam transmitir diagnósticos e prognósticos difíceis (GEOVANINI F e BRAZ M, 2013).

A comunicação poderá ser tanto terapêutica, realizada findando o benefício da família e a o auxílio no dia a dia de internação, quanto negativo, podendo comprometer a saúde física e mental e oferecer momentos traumáticos caso o paciente negue tratamento medicamentoso para sua enfermidade (MONTEIRO D e QUINTANA A, 2016).

Sendo assim, o novo Código de Ética Médica, introduzido no ordenamento pela Resolução CFM 2.217/2018, veda ao médico, em seu artigo 13, deixar de informar o paciente sobre as determinantes sociais, ambientais ou profissionais de sua doença. Também proíbe, no artigo 24 , que o profissional médico deixe de ofererecer ao paciente o direito de tomar sua decisão sobre sua doença ou bem-estar, ficando assim a critério do paciente se deseja receber ou não tratamento, tanto farmacológico quanto paliativo para sua doença (GAZZOLA LPL, et al., 2020).

Além disso, acredita-se que desde o início do tratamento e da doença até o resultado final, todas as informações devem estar inseridas no processo de comunicação entre médicos, pacientes e familiares, e passar pelas várias etapas vividas pelos pacientes. Desta forma, o significado da palavra comunicação foi ampliado, vinculando-o ao apoio contínuo dos profissionais e às ideias compartilhadas entre todas as pessoas relevantes. $\mathrm{O}$ ato de comunicar-se com o paciente e seus familiares sobre o prognóstico da doença avançada é, antes de tudo, uma decisão clínica, assim como muitos outros pressupostos diários da prática médica, exigindo do profissional a reflexão, o estudo e análise sobre os fatos enunciados (GEOVANINI F e BRAZ M, 2013). 


\section{A interprofissionalidade e a comunicação de más notícias}

O Centro de Terapia Intensiva (CTI) é considerado um local complexo por conta da gravidade clínica dos pacientes em que se encontram nesse local, das angústias e preocupações dos familiares, da emoção e da rotina de trabalho da equipe médica e multiprofissional e, também, da necessidade dos familiares precisarem tomar decisões complexas e difíceis sobre o tratamento do paciente que se encontra internado. Entretanto, a comunicação entre paciente, família e equipe é imprescindível para tomar as decisões clínicas para nortear corretamente o cuidado do paciente no CTI (GIBELLO J, et al., 2020).

Apesar da comunicação de más notícias ser uma função quase que exclusiva direcionada ao profissional médico, na realidade, geralmente a comunicação de más notícias tem sido feita também pelos enfermeiros junto à equipe multiprofissional, garantindo a integralidade do cuidado com pacientes e seus familiares, diminuindo os danos que estão envolvidos no processo. Por outro lado, sabe-se que os profissionais da saúde, apesar de notarem a importância de uma boa comunicação interpessoal, relatam dificuldades em realizá-las como recurso terapêutico na assistência, principalmente nas abordagens da comunicação das más notícias e final de vida (ALMEIDA DR e ARAUJO MC, 2020; GALVÃO MIZ, et al., 2017).

Sabe-se também, que além dos profissionais da área da saúde comunicarem as más notícias, os mesmos devem ofertar os Cuidados Paliativos, que são caracterizados como cuidados ativos e integrais sugeridos para pacientes com doença grave, progressiva e que ameace a continuidade de sua vida, bem como quando a doença não responde mais a tratamentos curativos, objetivando assim proporcionar conforto e melhoria da qualidade de vida destes indivíduos e de seus familiares, por meio do alívio do sofrimento, do tratamento da dor e dos sintomas e também intervenção em problemas biopsicossociais e espirituais. Esses cuidados objetivam a abordagem desde o diagnóstico da doença, prognóstico, até seus momentos finais, e continuando posteriormente para a família durante o luto. Todavia, para que essa assistência terapêutica tenha início de maneira eficaz e o mais precocemente possível, é crucial que haja estabelecimento de vínculos entre a equipe multiprofissional, o paciente e a sua família, além de uma comunicação feita de maneira adequada (EVANGELISTA CB, et al., 2017; BARROS BCS, et al., 2020).

Porém, como exemplo, pode-se utilizar no contexto da COVID-19. Percebe-se que a interprofissionalidade como meio modificador das práticas da equipe de saúde que envolve ações de comunicação eficaz, envolvimento na tomada de decisão, coesão, coordenação de cuidado, resolutividade de problemas e na decisão de paliativar o paciente quando necessário, sendo reconhecido que a falta desses fatores diminui a efetividade dos resultados (BELARMINO AC, et al., 2020).

Ademais, tais reflexões provam a importância da transmissão de más notícias ser fato compartilhado não apenas por quem atua na saúde ou em outras áreas relacionadas, mas sim por toda a sociedade. Nota-se que a comunicação de más notícias associa-se aos acontecimentos que podem alterar negativa, parcial ou drasticamente o futuro da vida das pessoa envolvidas (paciente, família e comunidade). Por conseguinte, destaca-se a importância de haver qualidade no processo de comunicação de más notícias por todos os profissionais que fazem parte da equipe multidisciplinar de saúde, desde o nível mais básico de atenção até o de maior complexidade de atendimento à população (GOMES ALZ e OTHERO MB, 2016; FONTES CMB, et al., 2017).

\section{CONSIDERAÇÕES FINAIS}

Em suma, infere-se que a comunicação de más notícias é uma das atividades mais difíceis e complexas exercidas pelos profissionais de saúde, exigindo treinamento e aperfeiçoamento. Por isso, há a necessidade de aprimorar a grade curricular das faculdades, com enfoque na transmissão das más notícias. Além do mais, o médico precisa aprender a lidar com a questão do emocional, não levando para o lado profissional a sensação do fracasso, e também aprender a lidar com a morte no cenário infantil, que poderiam ser enfrentadas através de terapias que auxiliam no preparo emocional dos profissionais da saúde. Em relação ao medo das emoções manifestadas pelo paciente, vê-se necessidade de adequar aos cursos de medicina 0 estudo da comunicação, especialmente para saber lidar com pacientes que possuem alguma patologia. 


\section{REFERÊNCIAS}

1. ALMEIDA DR, ARAUJO MC. Avaliação da aplicabilidade do protocolo spikes na comunicação de más notícias pelo profissional de enfermagem, 2020.

2. BARROS BCS, et al. As dificuldades enfrentadas na comunicação de más notícias pela equipe de cuidados paliativos: uma revisão. VII congresso internacional de envelhecimento humano, 2020.

3. BELARMINO AC, et al. Práticas colaborativas em equipe de saúde diante da pandemia de COVID-19. Revista brasileira de enfermagem, 2020; 73(6).

4. CAMPOS CACA, et al. Desafios da comunicação em Unidade de Terapia Intensiva Neonatal para profissionais e usuários. Revista Saúde em Debate, 2020; 41(2).

5. CRUZ CO, RIERA R. Comunicando más notícias: o protocolo SPIKES. Biblioteca Virtual em Saúde, 2016; 21(3).

6. EVANGELISTA CB, et al. Cuidados paliativos e espiritualidade: revisão integrativa da literatura. Revista brasileira de enfermagem, 2016; 69(3).

7. FONTES CMB, et al. Comunicação de más notícias: revisão integrativa de literatura na enfermagem. Revista brasileira de enfermagem, 2017; 70(5).

8. FREIBERGER MH, et al. Comunicação de más notícias a pacientes na perspectiva de estudantes de medicina. Revista Bioética, 2019; 27(2).

9. GALVÃO MIZ, et al. Comunicação interpessoal com pacientes oncológicos em cuidados paliativos. Revista baiana enfermagem, 2017; 31(3).

10. GAZZOLA LPL, et al. Comunicando más notícias sobre malformações congênitas: reflexões bioéticas e jurídicas. Revista bioética, 2020; 28(1).

11. GEOVANINI F, BRAZ M. Conflitos éticos na comunicação de más notícias em oncologia. Revista Bioética, 2013; 21(3).

12. GIBELLO J, et al. A. Importância da Comunicação de Más Notícias no Centro de Terapia Intensiva. Revista SBPH, $2020 ; 23(1)$.

13. GOMES ALZ, OTHERO MB. Cuidados paliativos. Revista Estudos Avançados, 2016; 30(88).

14. GULARTE NDG, et al. Abordando a Relação Clínica e a Comunicação de Notícias Difíceis com o Auxílio das Artes e dos Relatos Vivos. Revista Brasileira de Educação Médica, 2019; 43(4).

15. $\mathrm{KOCH} \mathrm{CL}$, et al. Más notícias: significados atribuídos na prática assistencial neonatal/pediátrica. Revista Bioética, 2017; 25(3).

16. LYSAKOWSKI S, et al. Comunicação da morte em tempos de pandemia por covid-19: relato de experiência. Saberes Pleurais: Educação na Saúde, 2020; 4(2).

17. MONTEIRO DT, QUINTANA AM. A comunicação de más notícias na uti: perspectiva dos médicos. Psicologia: Teoria e Pesquisa, 2017; 32(4).

18. OSTERMANN AC, et al. Perspectivas otimistas na comunicação de notícias difíceis sobre a formação fetal. Cadernos de Saúde Pública, 2017; 33(8).

19. SANTOS GFATF, et al. Cuidados Paliativos em Oncologia: Vivência de Enfermeiros ao Cuidar de Crianças em Fase Final da Vida. Revista online de Pesquisa: Cuidado é Fundamental, 2020; 12.

20. VOGEL KP, et al. Comunicação de más notícias: ferramenta essencial na graduação médica. Revista Brasileira de Educação médica, 2020; 43(1). 